\title{
Status of use of protease inhibitors for the prevention and treatment of pancreatitis after endoscopic retrograde cholangiopancreatography: An epidemiologic analysis of the evidence-practice gap using a health insurance claims database
}

\author{
Takeshi Seta ${ }^{1}$, Yoshimitsu Takahashi ${ }^{2}$, Yukitaka Yamashita ${ }^{1}$, Takeo Nakayama ${ }^{2, *}$ \\ 'Department of Gastroenterology and Hepatology, Japanese Red Cross Society Wakayama Medical Center, Wakayama, Japan, \\ ${ }^{2}$ Department of Health Informatics, Kyoto University School of Public Health, Kyoto, Japan.
}

\begin{abstract}
Summary $\quad$ Existing evidence suggests that protease inhibitors (PIs) used to prevent or treat pancreatitis after endoscopic retrograde cholangiopancreatography (ERCP) are ineffective, and their use is not recommended by clinical practice guidelines. However, in Japan, PIs are administered with the aim to prevent or treat post-ERCP pancreatitis. This study aimed to clarify the gap between guideline recommendations and contents of practice. We used the health insurance claims database of Japan Medical Data Center. Among patients who had undergone ERCP, those with acute pancreatitis or post-ERCP pancreatitis recorded in claims as disease names were defined as post-ERCP pancreatitis patients. The study period was divided into three terms according to the date of publication of clinical practice guidelines for acute pancreatitis. Among 2,945 patients who had undergone ERCP, 2,847 were eligible for analysis. Of these, $1,375(48.3 \%)$ patients had claims with pancreatitis recorded as the disease name; PIs were prescribed to $1,238(\mathbf{9 0 . 0} \%)$. Rates of prescription of PIs were $72.3 \%$ in $2005-07,70.9 \%$ in 2008-09, and $83.6 \%$ in 2010-15, showing a significant increase $(p<0.001)$. In conclusion, PIs are administered in clinical practice in Japan for the purpose of preventing or treating pancreatitis, with an increasing trend in prescription in recent years.
\end{abstract}

Keywords: Protease inhibitor, pancreatitis, endoscopic retrograde cholangiopancreatography (ERCP), evidence-practice gap, health insurance claims database

\section{Introduction}

Since 1991, evidence-based medicine (EBM) has been adopted in various clinical fields along with an increase in social awareness that seeks high-quality medical care (1). However, it is not always the case that evidencebased medical care is practiced in clinical settings (27 ). One study reported that guideline-recommended medical care is provided to roughly $50 \%$ of adult patients (8). In Japan, rates of implementation of some guideline-recommended treatments are reported to be roughly $25 \%(3,7,9)$. Even results from multiple

\footnotetext{
*Address correspondence to:

Dr. Takeo Nakayama, Department of Health Informatics, Kyoto University School of Public Health, Yoshida-Konoe, Sakyo-ku, City of Kyoto, Kyoto 606-8501, Japan.

E-mail: nakayama.takeo.4a@kyoto-u.ac.jp
}

randomized controlled trials (RCTs) have gained little attention among general clinicians; in fact, those results are not necessarily reflected in actual clinical activities (10). In other words, there is an "evidence-practice gap." In order to fill this gap, active communication between clinicians and clinical researchers, i.e., those who create evidence, is necessary (11).

At present, several RCTs (12-14) and systematic reviews (15-17) have concluded that the administration of protease inhibitors (PIs) prior to endoscopic retrograde cholangiopancreatography (ERCP) has no preventive effect against post-ERCP pancreatitis. The first edition of the Japanese (JPN) Guidelines for the Management of Acute Pancreatitis (hereafter, "JPN guidelines"), issued in 2003 by the Japanese Society for Abdominal Emergency Medicine and Japan Pancreas Society (18), states that clinical usefulness of PIs in mild or moderate acute pancreatitis is unclear. 
Nonetheless, PIs are widely used in Japan (19). The third edition of the JPN guidelines, which was developed by the Japanese Society for Abdominal Emergency Medicine, Research Committee on Intractable Diseases of Pancreas supported by Health and Labour Sciences Research Grants of the Ministry of Health, Labour and Welfare of Japan, Japanese Society of Hepato-Biliary-Pancreatic Surgery, Japan Pancreas Society, and Japan Radiological Society, states that administration of PIs for acute pancreatitis could be considered an option, albeit on insufficient grounds (20). Moreover, the fourth edition states that the effectiveness of PIs for "improving the prognosis and rate of complications associated with acute pancreatitis has not been clearly proven" by clinical studies and avoiding routine use is suggested (21). Furthermore, according to this edition, active evidence suggests a lack of efficacy of PIs administered prior to ERCP for the purpose of preventing the onset of post-ERCP pancreatitis (21). However, updated data from observational studies of actual clinical settings suggest the limited use of evidence $(19,22)$. In 2015, the Japan Pancreas Society published clinical practice guidelines for post-ERCP pancreatitis (23), which did not recommend the use of PI because it does not prevent post-ERCP pancreatitis (5) based on several RCTs $(13,14)$ and meta-analyses $(15,16)$. However, it also states, without citation, that PIs - which are covered by insurance - are generally administered as a matter of principle if patients are diagnosed with acute pancreatitis.

Based on the evidence currently available, the value of PIs for treating acute pancreatitis or preventing the development of post-ERCP pancreatitis is low. From the viewpoint of a clinician, however, it is difficult to judge whether or not to prescribe PIs, since descriptions regarding their use in clinical practice guidelines are inconsistent. This study aimed to investigate the status of PI use for preventing or treating post-ERCP pancreatitis in Japan and clarify factors associated with the gap between guideline recommendations and actual clinical practice.

\section{Materials and Methods}

\subsection{Study design}

Japan has a universal health insurance system, and healthcare services are provided through public insurers largely divided into the following three categories: Employee Health Insurance, National Health Insurance administered by local governments, and the Medical Care System for the Advanced Elderly (aged $\geq$ 75 years). Japanese citizens (i.e., the insured) pay insurance premiums to their medical insurers, and when they receive medical services from authorized insurance medical institutions such as hospitals, clinics, and pharmacies, they accept responsibility for some costs, while the remaining expenses are covered by payments of medical service fees made by the medical insurer through the examination and payment agency. All residents of Japan are required to subscribe to either one of the public insurances, and their activities (i.e., medical services they receive) can be traced, unless they cancel their insurance, no matter where or how many medical institutions/pharmacies they visit.

The present study used data registered from February 2005 through January 2015 (extracted in August 2015) in the Japan Medical Data Center (JMDC) Claims Database (developed by the Japan Medical Data Center Co., Ltd.). While the JMDC Claims Database only contains data of company employees (aged $\leq 74$ years) and their families, they can be used to examine disease prevalence and incidence in this population by matching claims data with health insurance enrollment data. Moreover, health insurance claims of all enrolled individuals are aggregated by name in an anonymized state and are thus traceable, even if they transfer to another hospital or visit multiple facilities. Thus, the actual state of medical services provided, not only by large-scale medical facilities but also medium-sized to small hospitals, as well as medical clinics with beds, can be assessed (23). This allows us to understand better the true state of real-world medical care in a continuous fashion, as the database provides complete data (i.e., administrative data) of all individuals enrolled in health insurance unions (24).

\subsection{Eligibility criteria}

Patients who underwent ERCP were defined as those for whom ERCP was performed with or without subsequent biliary or pancreatic procedures (procedures 1 and 2; Table 1). Among these, those with "acute pancreatitis" (disease code: K85) or "post-ERCP pancreatitis" (disease code: K918 or K85) as recorded in claims were defined as patients with post-ERCP pancreatitis and were included in this study.

\subsection{Data items and extraction methods}

Sex, age, number of beds, date of claims, type of PI used (by generic name), and the method of PI administration (intravenous drip infusion) were used as patient factors. Among the names of diseases recorded in claims, we could not distinguish between severe acute pancreatitis and acute pancreatitis, as the same disease code (K85) was used for both. Therefore, when claims containing the disease name "acute pancreatitis" or "post-ERCP pancreatitis" also contained at least one of the severe disorders listed in Table 2, these patients were assumed to be severe acute pancreatitis patients. Given the possibility that the publication of clinical practice guidelines might have altered treatment practice, analyses were performed by dividing the study period 
Table 1. Specific codes for ERCP examination and procedures

\begin{tabular}{|c|c|c|}
\hline Procedure & Name & Specific code \\
\hline 1 & Endoscopic retrograde cholangiopancreatography & $\begin{array}{l}\text { D308 + (cholangiography or pancreatography or } \\
\text { cholangiopancreatography) }\end{array}$ \\
\hline \multirow[t]{8}{*}{2} & Endoscopic nasobiliary drainage & K682-3 \\
\hline & Endoscopic cholangiography and stone removal & K685 or K6851 or K6852 \\
\hline & Endoscopic biliary balloon dilation & K686 \\
\hline & Endoscopic papillotomy & K687 or K6871 or K6872 \\
\hline & Endoscopic biliary stenting & K688 \\
\hline & Endoscopic pancreatic stenting & K708-3 \\
\hline & Endoscopic pancreatic pseudocyst drainage & K7071 \\
\hline & Endoscopic pancreatic stone removal (transduodenal sphincterotomy) & K6992 \\
\hline
\end{tabular}

ERCP, endoscopic retrograde cholangiopancreatography.

Table 2. Indicators for suspected (presumed) severe acute pancreatitis patients

\begin{tabular}{ll}
\hline Name & Specific code \\
\hline Maintenance or sustained dialysis & J038 or J038-2 \\
Artificial respiration & J045 \\
Angiography & E002 \\
Abdominal paracentesis & J010 or J021 \\
Disseminated intravascular coagulation & D65 \\
Nasoenteric feeding & J120 \\
Use of blood products & K920 \\
Organ failure & R688 \\
Shock & A419 or R571 or R579 or R570 \\
Respiratory failure & J960 or J969 or R090 \\
Renal failure & N170 or N178 or N179 or N19 \\
Gastrointestinal hemorrhage & K921 or K922 \\
\hline
\end{tabular}

into the first (2003-2007), second (2008-2009), and third (2010-2015) terms, according to the year of publication of the first (2003), second (2007), third (2009), and fourth (2015) editions. The identification code (ID) of each medical institution and hospital size (number of beds) were extracted as medical institution factors. Specifically, usage status was assessed according to the number of beds of $<200, \geq 200$ and $<500$, and $\geq 500$.

\subsection{Statistical analysis}

Proportions of patients for whom PIs were used were determined for those who developed post-ERCP pancreatitis and those who did not. Univariate analyses (Chi-square test, etc.) were performed by patient factors and severity. Subgroup analyses were performed to examine whether the trend for PI use differs by time of practice (i.e., first, second, and third terms) and number of beds, and trends were examined by the Cochrane-Armitage test. To examine factors associated with PI use, multiple logistic regression analysis was performed with severe patients (Model 1), post-ERCP pancreatitis (Model 2), and ERCP procedure (Model 3 ) as explanatory variables, in addition to sex, age, and number of beds. Moreover, the variance inflation factor (VIF) was calculated to examine multicollinearity of variables, and goodness-of-fit of each model was evaluated with the Hosmer-Lemeshow test. All tests were two-tailed, and $p<0.05$ was considered statistically significant. All analyses were performed using Stata/SE 14 (StataCorp LLC, TX, USA) (25).

\subsection{Ethical considerations}

This study was approved by the Ethics Committee of Kyoto University Graduate School and Faculty of Medicine (Approval No. R 0838) and the Ethics Committee of Japanese Red Cross Wakayama Medical Center (No. 447).

\section{Results}

\subsection{Patient characteristics}

Among 3,204,575 insured individuals who comprised the observed population, 2,945 had undergone ERCP (1,897 men and 1,048 women; median age, 55 years) (Figure 1). Of these, 2,847 patients were subjected to analysis, excluding 98 who were considered ineligible due to missing data.

Among the 2,847 analyzed patients, 1,375 (48.3\%) developed post-ERCP pancreatitis, while the remaining $1,472(51.7 \%)$ did not. PIs were used in $1,238(90.0 \%)$ of the 1,375 patients with post-ERCP pancreatitis, and $1,083(73.6 \%)$ of the 1,472 patients without post-ERCP pancreatitis. Among those for whom PIs were used, 


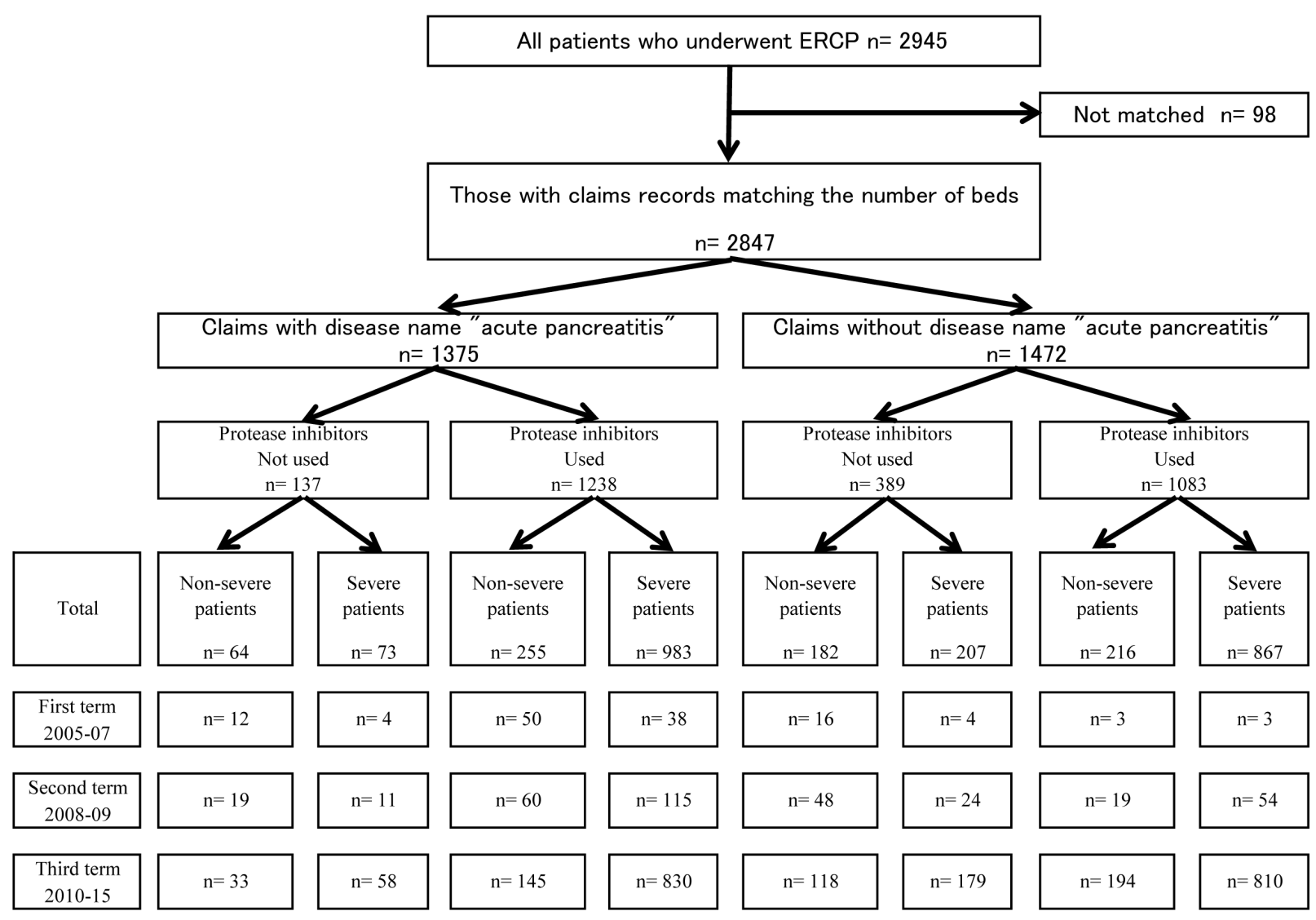

Figure 1. Relationships between patients who underwent ERCP, status of protease inhibitor use, and severity.

$225(20.6 \%)$ of the 1,238 patients with post-ERCP pancreatitis were non-severe patients, and $216(19.9 \%)$ of the 1,083 patients without post-ERCP pancreatitis were non-severe patients.

\subsection{Analysis results of the frequencies of protease inhibitors using to prevent and treat pancreatitis after ERCP and multiple logistic regression analysis}

Table 3 shows the number of patients who underwent ERCP and changes in the number of PI prescriptions over time. The proportions of PI users for the first, second, and third terms according to the year of publication of the JPN guidelines were $72.3 \%, 70.9 \%$, and $83.6 \%$, respectively, showing a significant increase over time $(p<0.001)$, with a particularly marked increase from the second to the third term. Among severe patients, the proportions of PI users were $83.7 \%$, $82.8 \%$, and $87.4 \%$, respectively, and although no significant increasing trend was observed $(p=0.15)$, the proportions of PI users remained high throughout the entire period. When the analysis was limited to nonsevere patients, the proportions of PI users for each term were $65.4 \%, 54.1 \%$, and $69.2 \%$, respectively, with a significant increasing trend over time $(p=0.003)$, particularly from the second to the third term.

Table 4 shows the results of subgroup analysis of patients with post-ERCP pancreatitis. The rate of PI use significantly increased over time $(p=0.004)$. When the analysis was limited to severe or non-severe patients, an increase in the rate of PI use was observed, but changes were not significant $(p=0.52$ and $p=0.59$, respectively).

Table 5 shows the results of the subgroup analysis of patients without post-ERCP pancreatitis. The rate of PI use significantly increased over time $(p<0.001)$. When limited to severe or non-severe patients, the analysis revealed a significant increase in the rate of PI use in both severe and non-severe patients $(p=0.001$ and $p<0.001$, respectively).

Table 6 shows the proportion of PI users by hospital/clinic size. The proportion of PI users and severity showed a significant decreasing trend with an increasing number of beds.

Table 7 shows the results of multiple logistic regression analysis. In Model 1, the rate of PIs use was significantly more frequent among severe patients (odds ratio: 3.48 [95\% confidence interval: 2.85-4.25]). In addition, the rate of PIs use was significantly less frequent at facilities with a higher number of beds. While the presence of post-ERCP pancreatitis was significantly associated with PI use (Model 2), no association was observed with ERCP procedure (Model $3)$. The results of the Hosmer-Lemeshow test were $p$ $=0.07, p=0.21$, and $p=0.01$ for Models 1, 2, and 3, respectively, with the highest VIF of 2.09. 
Table 3. Number of patients who underwent ERCP and changes in the number of protease inhibitor prescriptions over time (overall)

\begin{tabular}{lccc}
\hline Items & Overall & Severe patients & Non-severe patients \\
\hline First term (2005-2007) & & & 81 \\
$\quad$ No. patients undergoing ERCP & 130 & 49 & 53 \\
$\quad$ No. PIs prescribed & 94 & 41 & 65.4 \\
$\quad$ Proportion (\%) & 72.3 & 83.7 & 146 \\
Second term (2008-2009) & 350 & 204 & 79 \\
$\quad$ No. patients undergoing ERCP & 248 & 169 & 54.1 \\
$\quad$ No. PIs prescribed & 70.9 & 82.8 & 490 \\
$\quad$ Proportion (\%) & 2,367 & 1,877 & 339 \\
Third term (2010-2011) & 1,979 & 1,640 & 69.2 \\
$\quad$ No. patients undergoing ERCP & 83.6 & 87.4 & 717 \\
$\quad$ No. PIs prescribed & & & 471 \\
$\quad$ Proportion (\%) & 2,847 & 2,130 & 65.7 \\
Total & 2,321 & 1,850 & 0.003 \\
$\quad$ No. patients undergoing ERCP & 81.5 & 86.9 & \\
$\quad$ No. PIs prescribed & $<0.001$ & 0.15 & \\
$\quad$ Proportion (\%) & & & \\
value for trend & & & \\
\hline
\end{tabular}

ERCP, Endoscopic retrograde cholangiopancreatography; PI, Protease inhibitors.

Table 4. Number of patients who underwent ERCP and changes in the number of protease inhibitor prescriptions over time (with post-ERCP pancreatitis)

\begin{tabular}{|c|c|c|c|}
\hline Items & Overall & Severe patients & Non-severe patients \\
\hline \multicolumn{4}{|l|}{ First term (2005-2007) } \\
\hline No. patients undergoing ERCP & 104 & 42 & 62 \\
\hline No. PIs prescribed & 88 & 38 & 50 \\
\hline Proportion (\%) & 84.6 & 90.5 & 80.6 \\
\hline \multicolumn{4}{|l|}{ Second term (2008-2009) } \\
\hline No. patients undergoing ERCP & 205 & 126 & 79 \\
\hline No. PIs prescribed & 175 & 115 & 60 \\
\hline Proportion (\%) & 85.4 & 91.3 & 75.9 \\
\hline \multicolumn{4}{|l|}{ Third term (2010-2011) } \\
\hline No. patients undergoing ERCP & 1,066 & 888 & 178 \\
\hline No. PIs prescribed & 975 & 830 & 145 \\
\hline Proportion (\%) & 91.5 & 93.5 & 81.5 \\
\hline \multicolumn{4}{|l|}{ Total } \\
\hline No. patients undergoing ERCP & 1,375 & 1,056 & 319 \\
\hline No. PIs prescribed & 1,238 & 983 & 255 \\
\hline Proportion (\%) & 90.0 & 93.1 & 79.9 \\
\hline$P$ value for trend & 0.004 & 0.52 & 0.59 \\
\hline
\end{tabular}

ERCP, Endoscopic retrograde cholangiopancreatography; PI, Protease inhibitors.

Table 5. Number of patients who underwent ERCP and changes in the number of protease inhibitor prescriptions over time (without post-ERCP pancreatitis)

\begin{tabular}{|c|c|c|c|}
\hline Items & Overall & Severe patients & Non-severe patients \\
\hline \multicolumn{4}{|l|}{ First term $(2005-2007)$} \\
\hline No. patients undergoing ERCP & 26 & 7 & 19 \\
\hline No. PIs prescribed & 6 & 3 & 3 \\
\hline Proportion $(\%)$ & 23.1 & 42.9 & 15.8 \\
\hline \multicolumn{4}{|l|}{ Second term (2008-2009) } \\
\hline No. patients undergoing ERCP & 145 & 78 & 67 \\
\hline No. PIs prescribed & 73 & 54 & 19 \\
\hline Proportion (\%) & 50.3 & 69.2 & 28.4 \\
\hline \multicolumn{4}{|l|}{ Third term $(2010-2011)$} \\
\hline No. patients undergoing ERCP & 1,301 & 989 & 312 \\
\hline No. PIs prescribed & 1,004 & 810 & 194 \\
\hline Proportion (\%) & 77.2 & 81.9 & 62.2 \\
\hline \multicolumn{4}{|l|}{ Total } \\
\hline No. patients undergoing ERCP & 1,472 & 1,074 & 398 \\
\hline No. PIs prescribed & 1,083 & 867 & 216 \\
\hline Proportion $(\%)$ & 73.6 & 80.7 & 54.3 \\
\hline$P$ value for trend & $<0.001$ & 0.001 & $<0.001$ \\
\hline
\end{tabular}

ERCP, Endoscopic retrograde cholangiopancreatography; PI, Protease inhibitors. 
Table 6. Relationship between hospital/clinic size and the number of protease inhibitor users

\begin{tabular}{|c|c|c|c|c|c|}
\hline $\begin{array}{l}\text { Hospital/ } \\
\text { clinic size }\end{array}$ & $\begin{array}{l}\text { Total number } \\
\text { of patients }\end{array}$ & $\begin{array}{l}\text { No. patients who used } \\
\text { protease inhibitors }(\%)\end{array}$ & $\begin{array}{l}\text { No. of patients with } \\
\text { severe pancreatitis }\end{array}$ & $\begin{array}{l}\text { No. of patients who used } \\
\text { protease inhibitors with } \\
\text { severe pancreatitis }(\%)\end{array}$ & $\begin{array}{l}\text { Percentage of patients with } \\
\text { severe pancreatitis to total } \\
\text { number of patients }\end{array}$ \\
\hline$\leq 199$ & 209 & $186(89.0)$ & 163 & $145(89.0)$ & 78.0 \\
\hline $200-499$ & 873 & $696(79.7)$ & 658 & $558(84.8)$ & 75.4 \\
\hline$\geq 500$ & 1,765 & $1,439(81.5)$ & 1,309 & $1,147(87.6)$ & 74.2 \\
\hline Total & 2,847 & $2,321(81.5)$ & 2,130 & $1,850(86.9)$ & 74.8 \\
\hline
\end{tabular}

Table 7. Results of multiple logistic regression analysis on protease inhibitor use

\begin{tabular}{|c|c|c|c|c|c|c|c|c|c|}
\hline \multirow{3}{*}{ Explanatory variables } & \multicolumn{3}{|c|}{ Model 1} & \multicolumn{3}{|c|}{ Model 2} & \multicolumn{3}{|c|}{ Model 3} \\
\hline & \multirow{2}{*}{$\begin{array}{l}\text { Odds } \\
\text { Ratio }\end{array}$} & \multicolumn{2}{|c|}{ 95\% Confidence Interval } & \multirow{2}{*}{$\begin{array}{l}\text { Odds } \\
\text { Ratio }\end{array}$} & \multicolumn{2}{|c|}{ 95\% Confidence Interval } & \multirow{2}{*}{$\begin{array}{l}\text { Odds } \\
\text { Ratio }\end{array}$} & \multicolumn{2}{|c|}{$95 \%$ Confidence Interval } \\
\hline & & Lower limit & Upper limit & & Lower limit & Upper limit & & Lower limit & Upper limit \\
\hline Severe patients & 3.48 & 2.85 & 4.25 & & N/A & & & N/A & \\
\hline Post-ERCP pancreatitis & & N/A & & 3.26 & 2.62 & 4.04 & & N/A & \\
\hline ERCP procedure & & N/A & & & N/A & & 1.22 & 0.99 & 1.50 \\
\hline Sex & 1.10 & 0.89 & 1.35 & 1.11 & 0.90 & 1.36 & 1.09 & 0.89 & 1.34 \\
\hline Age & 1.00 & 0.99 & 1.00 & 1.00 & 0.99 & 1.00 & 0.99 & 0.99 & 1.00 \\
\hline \multicolumn{10}{|l|}{ No. beds } \\
\hline$\leq 199$ & \multicolumn{3}{|c|}{1.00 (Reference) } & \multicolumn{3}{|c|}{1.00 (Reference) } & \multicolumn{3}{|c|}{1.00 (Reference) } \\
\hline $200-499$ & 0.50 & 0.31 & 0.80 & 0.67 & 0.42 & 1.08 & 0.48 & 0.30 & 0.76 \\
\hline$\geq 500$ & 0.57 & 0.36 & 0.90 & 0.86 & 0.54 & 1.37 & 0.54 & 0.34 & 0.84 \\
\hline Hosmer-Lemeshow test & & $P=0.07$ & & & $P=0.21$ & & & $P=0.01$ & \\
\hline
\end{tabular}

N/A, Not applicable; ERCP, Endoscopic retrograde cholangiopancreatography.

\section{Discussion}

Existing evidence suggests that PIs used to treat patients with acute pancreatitis, or to treat or prevent the onset of post-ERCP pancreatitis, is ineffective. While such use is also not recommended by clinical practice guidelines, we found that $80 \%$ of patients who had undergone ERCP, and $65 \%$ of non-severe acute pancreatitis patients, were administered PIs in Japan. This is the first report to show the actual state of PI prescription related to ERCP using large-scale realworld data.

Sekimoto et al. conducted a questionnaire survey on clinical practice for acute pancreatitis among clinicians before and after publication of the JPN guidelines, and reported that the use of PIs for patients with mild pancreatitis significantly decreased after publication of the guidelines at the time; no such change was observed in the use of PIs for moderate to severe pancreatitis (19). Murata et al. showed that intravenous PI infusion was administered in $86 \%$ and $80 \%$ of patients with mild acute pancreatitis before and after publication of the first (2003) edition of the JPN guidelines, respectively, using the Diagnosis Procedure Combination (DPC) system (22). Moreover, around the time when the third (2009) edition was published, PIs were used in $60.8 \%$ of patients with mild pancreatitis, and while the frequency of use had decreased since the publication of the first edition, $82.9 \%$ of patients - when considering those with severe pancreatitis only - were using PIs (22). Based on these results, Murata et al. concluded that there has been no change in clinical practice policy among clinicians in the past few years, and PIs are still used according to the traditional philosophy. The results of these previous studies, which used different methods to evaluate the degree of penetration of clinical practice guidelines, did not differ greatly from the results of the present study, which targeted a wider range of hospitals/clinics without limiting the analysis to DPC hospitals.

In the present study, we initially expected that the use of PIs for the prevention or treatment of pancreatitis occurring due to ERCP examination might have decreased with revisions of the JPN guidelines. However, in reality, PIs were used at a higher rate than expected even in the past few years. A possible reason for the high number of PI prescriptions is the existence of "clinical inertia" for the evidence-practice gap. It is said that physicians cannot easily change a traditional medical practice because they have been a human behavior, clinical inertia. Barth JH et al. showed "The main barriers include awareness, familiarity and agreement with the contents" as reasons why clinical practice guidelines cannot be adhered, and it is difficult to correct in the correct direction because familiar act exists (26).

The present study examined how the date of publication of clinical practice guidelines and the number of beds were related to the rate of PI use. With respect to the date of publication of clinical practice guidelines, the use of PIs did not decrease with guideline updates or dissemination, but rather showed an increasing trend. With respect to the number of beds, we hypothesized that the use of PIs might show 
a decreasing trend with an increasing number of beds, i.e., big hospitals with many acute patients, since there are not only many patients undergoing ERCP but also specialists who are highly interested in clinical practice guidelines. The results of the analysis revealed that, while the rate of PI use significantly decreased with an increasing number of beds, more than $80 \%$ of patients who had undergone ERCP were administered PIs. These findings suggest that the understanding of the JPN guidelines and implementation of the recommendations have not improved over time more than we had anticipated, irrespective of the hospital or clinic size.

While sex and age of patients did not contribute to PI use, a significantly higher use was observed among severe patients. Progression to a severe state is known to be triggered by the onset of post-ERCP pancreatitis following ERCP procedures (endoscopic papillotomy, endoscopic biliary stenting, or endoscopic pancreatic stenting), which by itself is associated with severe disease. Moreover, ERCP examination itself could cause post-ERCP pancreatitis, thereby advancing in severity (21). ERCP procedures and post-ERCP pancreatitis are thus likely to act as intermediate (or mediating) variables. In clinical studies, variables for regression analysis should be selected based on clinical judgments, since a misplaced sense of confidence in statistical methods could lead to the selection of clinically meaningless variables or overfitting of models (27). For these reasons, we considered Model 1 (severe patients) to be the most clinically meaningful. The results of the multiple logistic regression analysis revealed a trend of increased PI use in severe patients. The most recent version of the JPN guidelines recommends against the routine use of PIs, based on the evidence suggesting a lack of efficacy of PIs on acute pancreatitis (21). Moreover, the effect of PIs in patients with severe acute pancreatitis has been demonstrated in clinical studies with high bias risk (17), so the true effect of PIs remains unclear. The present study revealed an increasing trend in PI use among patients with post-ERCP pancreatitis and those with severe conditions, suggesting the possibility that JPN guideline recommendations relating to treatment have not fully permeated into the practice of clinicians.

There are some limitations to this study. First, the JMDC medical data bank targets individuals enrolled in health insurance unions for employees of large companies, i.e., a population comprising individuals of relatively high socioeconomic status. As the enrollees are mainly in their 30 s to 50 s, the number of cases in which ERCP examination was performed was low. Second, disease names recorded in claims data do not necessarily reflect the accurate names of diseases diagnosed. The method used in this study does not allow for distinction between patients with 'true' postERCP pancreatitis and those with acute pancreatitis 'recorded in claims as the disease name,' since this was assigned in order to prescribe PIs for the purpose of preventing the onset of post-ERCP pancreatitis. Third, as health insurance claims do not contain clinical information and examination data, we could not assess the severity of pancreatitis using these data (28). While the rate of post-ERCP pancreatitis advancing in severity is reportedly low at around 2\% (28), twothirds of patients in the present study were in severe condition, which was clearly high. However, regardless of the severity of pancreatitis, there is no evidence that supports the effectiveness of PIs, and the conclusion that these drugs are over-prescribed for pancreatitis in Japan stands firm.

In conclusion, the present study found that, despite that no evidence support effectiveness of PIs on postERCP pancreatitis prevention and treatment, and that clinical practice guidelines don't recommend the use, PIs are frequently prescribed to prevent or treat postERCP pancreatitis in Japan, with an increasing trend even in recent years. But the proportion of PI users and severity showed a significant decreasing trend with an increasing number of beds.

\section{Acknowledgements}

We sincerely thank Japan Medical Data Center Co., Ltd. for contributing their research data. Source of finding: The 2014-2015 Health and Labor Sciences Research Grant from the Ministry of Health, Labour and Welfare (research project for promoting the development of community healthcare infrastructure), "Research on the issues and possibilities of clinical practice guidelines as the foundation of healthcare that fulfills social responsibility." (Representative researcher: Takeo Nakayama).

\section{References}

1. Guyatt G. Evidence-based medicine. ACP J Club (Ann Intern Med). 1991; 114 (suppl 2): A-16.

2. Wada M, Nakayama T, Ishizaki T, Satoh T, Ikeda S. Practice patterns for lower respiratory tract infections in hospital patients with particular focus on bacteriological examinations and injection antibiotics use. Int J Gen Med. 2013; 6:597-604.

3. Kirigaya D, Nakayama T, Ishizaki T, Ikeda S, Satoh T. Management and treatment of osteoporosis in patients receiving long-term glucocorticoid treatment: current status of adherence to clinical guidelines and related factors. Intern Med. 2011; 50:2793-2800.

4. Nakaoka S, Ishizaki T, Urushihara H, Satoh T, Ikeda S, Yamamoto M, Nakayama T. Prescribing pattern of antiParkinson drugs in Japan: a trend analysis from 2005 to 2010. PLoS One. 2014; 9:e99021.

5. Kimura S, Sato T, Ikeda S, Noda M, Nakayama T. Development of a database of health insurance claims:standardization of disease classifications and anonymous record linkage. J Epidemiol. 2010; 20:413419. 
6. "Important Precautions" Revised information [internet]. Tokyo: Ministry of Health, Labour and Welfare; c2007. Available from: http://www.info.pmda.go.jp/kaitei/ kaitei20070419.html\#l (accessed June 26, 2019). (in Japanese)

7. Nakaoka S, Ishizaki T, Urushihara H, Satoh T, Ikeda S, Morikawa K, Nakayama T. Echocardiography for the detection of valvulopathy associated with the use of ergotderived dopamine agonists in patients with Parkinson's disease. Intern Med. 2011; 50:687-694.

8. McGlynn EA, Asch SM, Adams J, Keesey J, Hicks J, DeCristofaro A, Kerr EA. The quality of health care delivered to adults in the United States. N Engl J Med. 2003; 348:2635-2645.

9. Kanazawa N, Ueshima K, Tominari S, Nakayama T. Underuse of cardiac rehabilitation in workers with coronary artery disease - Claims database survey in Japan. Circ J. 2017; 81:1424-1431.

10. Evensen AE, Sanson-Fisher R, D'Este C, Fitzgerald M. Trends in publications regarding evidence-practice gaps: A literature review. Implement Sci. 2010; 5:11.

11. Teachman BA, Drabick DA, Hershenberg R, Vivian D, Wolfe BE, Goldfried MR. Bridging the gap between clinical research and clinical practice: introduction to the special section. Psychotherapy (Chic). 2012; 49:97-100.

12. Zheng M, Chen Y, Yang X, Li J, Zhang Y, Zeng Q. Gabexate in the prophylaxis of post-ERCP pancreatitis: A meta-analysis of randomized controlled trials. BMC Gastroenterol. 2007; 7:6.

13. Rudin D, Kiss A, Wetz RV, Sottile VM. Somatostatin and gabexate for post-endoscopic retrograde cholangiopancreatography pancreatitis prevention:metaanalysis of randomized placebo-controlled trials. J Gastroenterol Hepatol. 2007; 22:977-983.

14. Manes G, Ardizzone S, Lombardi G, Uomo G, Pieramico O, Porro GB. Efficacy of postprocedure administration of gabexate mesylate in the prevention of post-ERCP pancreatitis: a randomized, controlled, multicenter study. Gastrointest Endosc. 2007; 65:982-987.

15. Seta T, Noguchi Y. Protease inhibitors for preventing complications associated with ERCP: an updated metaanalysis. Gastrointest Endosc. 2011; 73:700-706.

16. Andriulli A, Leandro G, Federici T, Ippolito A, Forlano R, Iacobellis A, Annese V. Prophylactic administration of somatostatin or gabexate does not prevent pancreatitis after ERCP: An updated meta-analysis. Gastrointest Endosc. 2007; 65:624-632.

17. Seta T, Noguchi Y, Shikata S, Nakayama T. Treatment of acute pancreatitis with protease inhibitors administered through intravenous infusion: An updated systematic review and meta-analysis. BMC Gastroenterol. 2014;
14:102.

18. JPN guideline for the management of acute pancreatitis (Takada T, eds.). 1st ed., Kanehara, Tokyo, JPN, 2003; p. 73. (in Japanese)

19. Sekimoto M, Shikata S, Takada T, et al. Changes in management of acute pancreatitis before and after the publication of evidence-based practice guidelines in 2003. J Hepatobiliary Pancreat Sci. 2010; 17:17-23.

20. JPN guideline for the management of acute pancreatitis (Takada T, eds.). 3rd ed., Kanehara, Tokyo, JPN, 2009; p. 108. (in Japanese)

21. JPN guideline for the management of acute pancreatitis (Takada T, eds.). 4th ed., JPN Guidelines 2015, Kanehara, Tokyo, JPN, 2015; p. 135. (in Japanese)

22. Murata A, Matsuda S, Mayumi T, Yokoe M, Kuwabara K, Ichimiya Y, Fujino Y, Kubo T, Fujimori K, Horiguchi H. A descriptive study evaluating the circumstances of medical treatment for acute pancreatitis before publication of the new JPN guidelines based on the Japanese administrative database associated with the Diagnosis Procedure Combination system. J Hepatobiliary Pancreat Sci. 2011; 18:678-683.

23. Mine T, Akashi R, Itoh T, Kawaguchi Y, Sugano A, Kida M, Hanada T, Miyagawa H, Yamaguchi T, Morisane T. Guideline of Post-ERCP pancreatitis. Suizo. 2015; 30:541-584. Available from https://www.jstage.jst.go.jp/ article/suizo/30/4/30_539/_article/-char/ja/ (accessed June 26, 2019). (in Japanese)

24. Nakayama T. Approaching the "evidence-practice gap" in pharmaceutical risk management: Analysis of healthcare claim data. Yakugaku Zasshi. 2012; 132:549-554. (in Japanese)

25. StataCorp. 2015. Stata Statistical Software: Release 14. College Station, TX: Stata Corp LP.

26. Barth JH, Misra S, Aakre KM, Langlois MR, Watine J, Twomey PJ, Oosterhuis WP. Why are clinical practice guidelines not followed? Clin Chem Lab Med. 2016; 54:1133-1139.

27. Shintani A. Multivariate analysis - how to choose explanatory variables. Syukan-Igakukai-shimbun 2011. Available from http://www.igaku-shoin.co.jp/paperDetail. do?id=PA02949_04 (accessed June 26, 2019). (in Japanese)

28. Loperfido S, Angelini G, Benedetti G, Chilovi F, Costan F, De Berardinis F, De Bernardin M, Ederle A, Fina P, Fratton A. Major early complications from diagnostic and therapeutic ERCP: a prospective multicenter study. Gastrointest Endosc. 1998; 48:1-10.

(Received May 7, 2019; Revised June 28, 2019; Accepted June 29, 2019) 